\title{
Well-posedness of infinite-dimensional linear systems with nonlinear feedback
}

\author{
Anthony Hastira, Federico Califano ${ }^{\mathrm{b}}$, Hans Zwart ${ }^{\mathrm{c}, \mathrm{d}}$ \\ ${ }^{a}$ University of Namur, Department of Mathematics and Namur Institute for Complex Systems (naXys), Rempart de la vierge, 8, B-5000 \\ Namur, Belgium \\ ${ }^{b}$ University of Twente, Robotics and Mechatronics (RAM), P.O. Box 217, 7500 AE, Enschede, The Netherlands \\ ${ }^{c}$ University of Twente, Department of Applied Mathematics, P.O. Box 217, 7500 AE Enschede, The Netherlands \\ ${ }^{d}$ Eindhoven University of Technology, Department of Mechanical Engineering, P.O. Box 513, 5600 MB Eindhoven, The Netherlands
}

\begin{abstract}
We study existence of solutions, and in particular well-posedness, for a class of inhomogeneous, nonlinear partial differential equations (PDE's). The main idea is to use system theory to write the nonlinear PDE as a well-posed infinitedimensional linear system interconnected with a static nonlinearity. By a simple example, it is shown that in general well-posedness of the closed-loop system is not guaranteed. We show that well-posedness of the closed-loop system is guaranteed for linear systems whose input to output map is coercive for small times interconnected to monotone nonlinearities. This work generalizes the results presented in (Tucsnak and Weiss, 2014), where only globally Lipschitz continuous nonlinearities were considered. Furthermore, it is shown that a general class of linear port-Hamiltonian systems satisfies the conditions asked on the open-loop system. The result is applied to show well-posedness of a system consisting of a vibrating string with nonlinear damping at the boundary.
\end{abstract}

Keywords: Well-posedness - passive infinite-dimensional systems - nonlinear feedback - boundary feedback port-Hamiltonian systems - vibrating string - nonlinear damping

\section{Introduction}

The notion of well-posedness for infinite-dimensional linear systems has been much studied in the last years, see e.g. (Staffans, 2005; Tucsnak and Weiss, 2009). More recently, existence of solution and in particular well-posedness of nonlinear partial differential equations (PDE's), has been addressed using system theory, see (Zwart et al., 2013). In the survey (Tucsnak and Weiss, 2014), conditions for the well-posedness of infinitedimensional linear systems are provided in detail. In that work, also the case with static nonlinear feedback has been addressed for globally Lipschitz continuous nonlinearities.

The problem of well-posedness for only locally Lipschitz continuous nonlinearities has been considered in the discussion paper (Zwart et al., 2013), where some issues related to this open problem were addressed.

The paper (Augner, 2016) provides conditions on a nonlinear boundary feedback interconnected with a linear port-Hamiltonian system to determine a nonlinear contraction semigroup. Even if those nonlinearities comprise some classes of locally Lipschitz continuous functions, wellposedness in the sense of (Tucsnak and Weiss, 2014) is not addressed for the closed-loop system.

In this work, we introduce a more general class of closedloop well-posed systems composed of a well-posed linear

Email addresses: anthony.hastir@unamur.be (Anthony Hastir), f.califano@utwente.nl (Federico Califano), h.j.zwart@utwente.nl, h.j.zwart@tue.nl (Hans Zwart) infinite-dimensional system whose input to output map is coercive for small times interconnected with static and monotone nonlinear feedback, which includes the class of locally Lipschitz continuous functions considered in (Augner, 2016).

This paper is organized as follows. In Section 2, the necessary background is presented and a motivating example which introduces the problem is provided. In particular, we recall the notion of well-posedness, both for linear and nonlinear systems. Section 3 is dedicated to the statement and the proof of the main result. In Section 4 , it is shown that the assumptions required on the linear open-loop system are satisfied for an important class of port-Hamiltonian systems. The result is applied to show the well-posedness of a vibrating string with a nonlinear damper at the boundary. Section 6 contains conclusions and future work.

\section{Background and problem statement}

As said in the introduction we follow the idea of (Tucsnak and Weiss, 2014). That is, we consider an inhomogeneous, non-linear system as the interconnection of an inhomogeneous linear system with a static nonlinearity as depicted in Figure 1. Furthermore, it is assumed that the linear part, denoted by $\Sigma^{P}$ is well-posed, of which we recall the definition first.

Consider the linear system $\Sigma^{P}$, with input space $U$, state space $X$, and output space $Y$ (all real Hilbert spaces), 


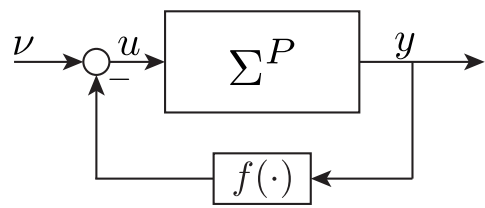

Figure 1: Representation of $\Sigma^{f}$.

described by the equations

$$
\Sigma^{P}:\left\{\begin{array}{l}
\dot{x}(t)=A x(t)+B u(t) \\
y(t)=C x(t)+D u(t) \\
x(0)=x_{0}
\end{array}\right.
$$

where $A, B, C$ and $D$ are in general unbounded operators.

Definition 2.1. The system $\Sigma^{P}$ is said to be well-posed if for every $u \in L_{l o c}^{2}([0, \infty) ; U)$ (input) and for every $x_{0} \in X$ (initial state), the abstract differential equation (1) possesses a unique (mild) solution $x \in \mathcal{C}([0, \infty) ; X)$ (state trajectory) and $y \in L_{l o c}^{2}([0, \infty) ; Y)$ (ouput function). Hence, if $\Sigma^{P}$ is well-posed, then the solution of (1) can be written using four families of bounded linear operators as follows: ${ }^{1}$

$$
\begin{aligned}
& x(t)=\mathbb{T}_{t} x_{0}+\Phi_{t} u, \\
& \mathbf{P}_{t} y=\Psi_{t} x_{0}+\mathbb{F}_{t} u,
\end{aligned}
$$

for all $t \in[0, \infty)$. Moreover, on any bounded time interval $[0, \tau], 0<\tau<\infty, x(\tau)$ and $\mathbf{P}_{\tau} y$ depend continuously on $x_{0}$ and on $\mathbf{P}_{\tau} u$.

Note that $\mathbb{T}_{t}$ is the $C_{0}$-semigroup generated by operator $A, \Phi_{t}$ is called the input map, $\Psi_{t}$ the output map, and $\mathbb{F}_{t}$ the input-output map, which satisfy the following properties, see (Tucsnak and Weiss, 2014):

- $\mathbb{T}=\left(\mathbb{T}_{t}\right)_{t \geq 0}$ is an operator $C_{0}$-semigroup on $X$,

- $\Phi=\left(\Phi_{t}\right)_{t \geq 0}$ is a family of bounded linear operators from $L^{2}([0, \infty) ; U)$ to $X$ such that

$$
\Phi_{\tau+t}(u \underset{\tau}{u} v)=\mathbb{T}_{t} \Phi_{\tau} u+\Phi_{t} v
$$

for every $u, v \in L^{2}([0, \infty) ; U)$ and all $\tau, t \geq 0$,

- $\Psi=\left(\Psi_{t}\right)_{t \geq 0}$ is a family of bounded linear operators from $X$ to $L^{2}([0, \infty) ; Y)$ such that

$$
\Psi_{\tau+t} x_{0}=\Psi_{\tau} x_{0}{ }_{\tau} \Psi_{t} \mathbb{T}_{\tau} x_{0},
$$

for every $x_{0} \in X$ and all $\tau, t \geq 0$ and $\Psi_{0}=0$,

- $\mathbb{F}=\left(\mathbb{F}_{t}\right)_{t \geq 0}$ is a family of bounded linear operators from $L^{2}([0, \infty) ; U)$ to $L^{2}([0, \infty) ; Y)$ such that

$$
\mathbb{F}_{\tau+t}(u \diamond v)=\mathbb{F}_{\tau} \underset{\tau}{\diamond}\left(\Psi_{t} \Phi_{\tau} u+\mathbb{F}_{t} v\right),
$$

for every $u, v \in L^{2}([0, \infty) ; U)$ and all $\tau, t \geq 0$, and $\mathbb{F}_{0}=0$,

\footnotetext{
${ }^{1}$ For a positive $t, \mathbf{P}_{t}$ denotes the operator of truncation to the interval $[0, t]$ of a function defined on a larger set than $[0, t]$, see (Tucsnak and Weiss, 2014).
}

where, for any $u, v \in L^{2}([0, \infty) ; U)$ and any $\tau \geq 0$, the $\tau$-concatenation of $u$ and $v$ is the function defined by

$$
u\rangle_{\tau} v=\mathbf{P}_{\tau} u+\mathbf{S}_{\tau} v
$$

Here $\mathbf{S}_{\tau}$ is defined as the bilateral right shift operator. With these notations, we denote $\Sigma^{P}=(\mathbb{T}, \Phi, \Psi, \mathbb{F})$. Moreover, these maps satisfy the following properties :

- $\Phi$ is causal, i.e., $\Phi_{\tau} \mathbf{P}_{\tau}=\Phi_{\tau}$ for all $\tau \geq 0$,

- For all $\tau, t \geq 0$,

$$
\begin{aligned}
\Phi_{\tau+t} \mathbf{P}_{\tau} & =\mathbb{T}_{t} \Phi_{\tau}, \\
\mathbf{P}_{\tau} \Psi_{\tau+t} & =\Psi_{\tau}, \\
\mathbf{P}_{\tau} \mathbb{F}_{\tau+t} \mathbf{P}_{\tau} & =\mathbf{P}_{\tau} \mathbb{F}_{\tau+t}=\mathbb{F}_{\tau} .
\end{aligned}
$$

The feedback interconnection of $\Sigma^{P}$ and $f$ as shown in Figure 1 is denoted by $\Sigma^{f}$ and is the dynamic system obtained by imposing :

$$
u(t)=\nu(t)-f(y(t)) \forall t \in[0, \infty) .
$$

Here we assume that $f: Y \rightarrow U$ is a static nonlinear continuous function and $\nu \in L_{l o c}^{2}([0, \infty) ; U)$ is the new external input.

Under the assumption that $\Sigma^{P}$ is well-posed, wellposedness for the nonlinear closed-loop system $\Sigma^{f}$ can be defined.

Definition 2.2. The closed-loop system $\Sigma^{f}$ is said to be well-posed if for any input $\nu \in L_{\text {loc }}^{2}([0, \infty) ; U)$ and any $x_{0} \in X$ (initial state) there exists $t_{f} \in(0, \infty]$ and unique functions $x \in \mathcal{C}\left(\left[0, t_{f}\right) ; X\right)$ (state trajectory) and $y \in L_{\text {loc }}^{2}\left(\left[0, t_{f}\right) ; Y\right)$ (output function) such that

$$
\begin{aligned}
& x(t)=\mathbb{T}_{t} x_{0}+\Phi_{t} \nu-\Phi_{t} f(y), \\
& \mathbf{P}_{t} y=\Psi_{t} x_{0}+\mathbb{F}_{t} \nu-\mathbb{F}_{t} f(y),
\end{aligned}
$$

for all $t<t_{f}$, and moreover, on any bounded time interval $[0, \tau], 0<\tau<t_{f}, x(\tau)$ and $\mathbf{P}_{\tau} y$ depend continuously on $x_{0}$ and on $\mathbf{P}_{\tau} \nu$.

In (Tucsnak and Weiss, 2014) is was shown that if $f$ is (globally) Lipschitz continuous and $\delta L<1$, (where $L$ is the Lipschitz constant of $f$ and $\left.\delta=\inf _{t>0}\left\|\mathbb{F}_{t}\right\|\right)$, then the closed-loop system is a well-posed system, and solutions exist globally, i.e., $t_{f}=\infty$. Based on this it is temping to believe that well-posedness with local existence of trajectories (i.e., $t_{f}<\infty$ ) will hold when $f$ is locally Lipschitz and $\delta=0$. The following example shows that this is false.

Example 2.1. As linear well-posed system we consider the controlled transport equation with observation, given by

$$
\begin{aligned}
\frac{\partial x}{\partial t}(\zeta, t) & =\frac{\partial x}{\partial \zeta}(\zeta, t), & & \zeta \in[0,1], t \geq 0 \\
x(\zeta, 0) & =x_{0}(\zeta), & & \zeta \in[0,1] \\
u(t) & =x(1, t), & & \geq 0 \\
y(t) & =x(0, t), & & t \geq 0 .
\end{aligned}
$$


As state space we choose $L^{2}([0,1] ; \mathbb{R})$. For this simple boundary control system the solution is given by

$$
x(\zeta, t)= \begin{cases}x_{0}(\zeta+t), & \zeta+t \leq 1 \\ u(\zeta+t-1), & \zeta+t>1\end{cases}
$$

and

$$
y(t)= \begin{cases}x_{0}(t), & t \leq 1 \\ u(t-1), & t>1\end{cases}
$$

Hence the system is clearly well-posed and $\delta=0$. Let us now consider the nonlinear feedback

$$
u(t)=-f(y(t))=y^{2}(t),
$$

as also depicted.

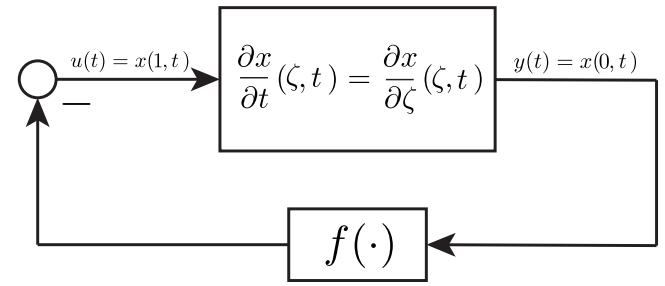

Figure 2: Nonlinear feedback interconnection of a pure shift and a static nonlinearity.

The solution for $t<1$ must take the form

$$
x(\zeta, t)= \begin{cases}x_{0}(\zeta+t), & \zeta+t \leq 1 \\ x_{0}^{2}(\zeta+t-1), & \zeta+t>1\end{cases}
$$

Consider now the following initial condition in $L^{2}([0,1] ; \mathbb{R})$

$$
x_{0}(\zeta)=\frac{1}{\sqrt[3]{\zeta}}
$$

For $0<t<1$ we have from (11)

$$
\begin{aligned}
\int_{0}^{1} x(\zeta, t)^{2} d \zeta & \geq \int_{1-t}^{1} x(\zeta, t)^{2} d \zeta \\
& =\int_{1-t}^{1} \frac{1}{\sqrt[3]{(\zeta+t-1)^{2}}} d \zeta=\infty
\end{aligned}
$$

Hence there does not exists any $t>0$ such that state lies in the state space, and so the system merged by this simple interconnection is not well-posed.

The above example implies that if we want/have to consider connection as in Figure 1 with $f$ (only) locally Lipschitz, then we have to impose extra condition on $\Sigma^{P}$ and $f$. In the following we assume $U$ and $Y$ to be the same real Hilbert space, i.e., $U=Y$. On the system we impose the following, where $\mathbb{F}_{t}$ was introduced in Definition 2.1.

Assumption 2.1. There exists $t^{*}>0$ such that for all $t<t^{*}$, the operator $\mathbb{F}_{t}$ is coercive ${ }^{2}$, i.e., there exists $\tilde{c}>0$ such that for all $u \in L^{2}\left(\left[0, t^{*}\right) ; U\right)$, it holds

$$
\left\langle\mathbb{F}_{t} u, u\right\rangle \geq \tilde{c}\langle u, u\rangle, \text { for all } t<t^{*}
$$

\footnotetext{
${ }^{2}$ Note that since $\mathbb{F}_{t}$ is coercive, it is boundedly invertible, see e.g. (Curtain and Zwart, 1995, Example A.4.2).
}

This condition can be interpreted as being strict input passive on small time intervals and for finite-dimensional systems it is satisfied if and only if $D+D^{T}>0$.

For the nonlinear function $f(\cdot)$ we assume the following.

Assumption 2.2. The nonlinearity satisfies the following properties:

- $f$ is continuous

- $\forall y_{1}, y_{2},\left\langle f\left(y_{1}\right)-f\left(y_{2}\right), y_{1}-y_{2}\right\rangle_{U} \geq 0$,

- $f(0)=0$.

Remark 2.1. The class of considered nonlinear functions $f(\cdot)$ comprises strictly increasing, positive and unbounded locally Lipschitz continuous (scalar) functions like odd polynomials (e.g. $\left.f(y)=y^{3}\right)$.

We end this section with a result on $m$-dissipativity.

Definition 2.3. The (nonlinear) operator $J$ on domain $D(J) \subset X$ is called $m$-dissipative if

- $J$ is dissipative, i.e., $\langle J x-J \tilde{x}, x-\tilde{x}\rangle_{X} \leq 0$ for $x, \tilde{x} \in$ $D(J)$;

- For all $\lambda>0$, the operator $J$ satisfies the range condition

$$
\begin{aligned}
X & =\{y \in X \mid \exists x \in D(J), y=(\lambda I-J)(x)\} \\
& =: \operatorname{Ran}(\lambda I-J) .
\end{aligned}
$$

Notice that since the operator $J$ is dissipative, the solution $x$ of the equation $(\lambda I-J)(x)=y$ for a given $y \in X$ and a given $\lambda>0$ is unique. In fact, suppose there are two solutions, $x_{1}$ and $x_{2}$, respectively. We have $y=\lambda x_{1}-J\left(x_{1}\right), y=\lambda x_{2}-J\left(x_{2}\right)$ so that

$$
\begin{aligned}
\lambda\left\|x_{1}-x_{2}\right\|^{2} & =\lambda\left\langle x_{1}-x_{2}, x_{1}-x_{2}\right\rangle \\
& =\left\langle J\left(x_{1}\right)-J\left(x_{2}\right), x_{1}-x_{2}\right\rangle \leq 0,
\end{aligned}
$$

which is possible if and only if $x_{1}=x_{2}$.

Lemma 2.1. Let $f: Y \mapsto Y$ be a function satisfying the conditions in Assumption 2.2, then for every $\lambda>0$ the range of $\lambda I+f$ equals $Y$, and thus $-f$ is $m$-dissipative. Furthermore,

$$
\|(\lambda I+f)(y)\| \geq \lambda\|y\| .
$$

Proof. Since the domain of $-f$ equals the whole space $Y$, it is maximally dissipative, i.e., it does not have a proper (dissipative) extension. Since $Y$ is a Hilbert space this gives that $-f$ is $m$-dissipative see (Miyadera, 1992, Section 2.3). For the norm inequality (12) we use the inequality in Assumption 2.2 with $y_{1}=y$ and $y_{2}=0$,

$$
\begin{aligned}
\|(\lambda I+f)(y)\|^{2}= & \langle(\lambda I+f)(y),(\lambda I+f)(y)\rangle \\
= & \lambda^{2}\|y\|^{2}+\lambda\langle y, f(y)\rangle+\lambda\langle f(y), y\rangle+ \\
& \|f(y)\|^{2} \geq \lambda^{2}\|y\|^{2} .
\end{aligned}
$$

Taking the square root on both sides ends the proof. 


\section{Main result}

First we state and prove some lemmas. For any continuous $f: U \rightarrow Y$ and any $t^{*}>0$, we define the operator $\Lambda_{f}$ by $\left(\Lambda_{f}(y)\right)(\cdot)=f(y(\cdot))$ for $y$ in

$D\left(\Lambda_{f}\right)=\left\{y \in L^{2}\left(\left[0, t^{*}\right) ; Y\right) \mid f(y(\cdot)) \in L^{2}\left(\left[0, t^{*}\right) ; U\right)\right\}$.

Since $D\left(\Lambda_{f}\right)=D\left(-\Lambda_{f}\right)$, the domain $D\left(\Lambda_{f}\right)$ will be used in the following.

Lemma 3.1. Under Assumption 2.2 the operator $-\Lambda_{f}$ on the domain $D\left(\Lambda_{f}\right)$ is $m$-dissipative.

Proof. Let first prove that $-\Lambda_{f}$ is dissipative. Taking $x, \tilde{x} \in D\left(\Lambda_{f}\right)$, we have

$\left\langle-\Lambda_{f}(x)-\left(-\Lambda_{f}(\tilde{x})\right), x-\tilde{x}\right\rangle=-\left\langle\Lambda_{f}(x)-\Lambda_{f}(\tilde{x}), x-\tilde{x}\right\rangle \leq 0$

since by assumption the last inequality holds pointwise.

It remains to prove that $\operatorname{Ran}\left(\lambda I-\left(-\Lambda_{f}\right)\right)=$ $L^{2}\left(\left[0, t^{*}\right) ; U\right)$ for all $\lambda>0$. So given $\lambda>0$, we have to show that for all $u \in L^{2}\left(\left[0, t^{*}\right) ; U\right)$, there exists $y \in D\left(\Lambda_{f}\right)$ such that $u=\left(\lambda I-\left(-\Lambda_{f}\right)\right)(y)$.

For $u \in L^{2}\left(\left[0, t^{*}\right) ; U\right)$, we define

$$
y(t)=(\lambda I+f)^{-1}(u(t)), \quad t \in\left[0, t^{*}\right)
$$

By Lemma 2.1 this inverse exists. Furthermore, using (12) we obtain that $y \in L^{2}\left(\left[0, t^{*}\right) ; U\right)$. Now since

$$
f(y(t))=(\lambda I+f)(y(t))-\lambda y(t)=u(t)-\lambda y(t)
$$

we find that $f(y(\cdot)) \in L^{2}\left(\left[0, t^{*}\right) ; U\right)$. Concluding, $-\Lambda_{f}$ is $m$-dissipative.

Lemma 3.2. Under Assumptions 2.1 and 2.2 the operator $\epsilon I-\mathbb{F}_{t^{*}}^{-1}-\Lambda_{f}$ on the domain $D\left(\epsilon I-\mathbb{F}_{t^{*}}^{-1}-\Lambda_{f}\right)=D\left(\Lambda_{f}\right)$ is dissipative for sufficiently small $\epsilon>0$.

Proof. By Assumption 2.1 it follows that $\mathbb{F}_{t^{*}}^{-1}$ exists and since $\mathbb{F}_{t^{*}}$ is coercive, $\mathbb{F}_{t^{*}}^{-1}$ is also coercive, i.e., there exists $c>0$ such that for all $y \in L^{2}\left(\left[0, t^{*}\right) ; Y\right)$, it holds $\left\langle\mathbb{F}_{t^{*}}^{-1} y, y\right\rangle \geq c\langle y, y\rangle$.

Let us now consider $y, \tilde{y} \in D\left(\epsilon I-\mathbb{F}_{t^{*}}^{-1}-\Lambda_{f}\right)$. It yields

$$
\begin{aligned}
& \left\langle\left(\epsilon I-\mathbb{F}_{t^{*}}^{-1}-\Lambda_{f}\right)(y)-\left(\epsilon I-\mathbb{F}_{t^{*}}^{-1}-\Lambda_{f}\right)(\tilde{y}), y-\tilde{y}\right\rangle \\
= & \left\langle-\Lambda_{f}(y)-\mathbb{F}_{t^{*}}^{-1} y+\epsilon y+\Lambda_{f}(\tilde{y})+\mathbb{F}_{t^{*}}^{-1} \tilde{y}-\epsilon \tilde{y}, y-\tilde{y}\right\rangle \\
= & -\left\langle\Lambda_{f}(y)-\Lambda_{f}(\tilde{y}), y-\tilde{y}\right\rangle-\left\langle\mathbb{F}_{t^{*}}^{-1}(y-\tilde{y}), y-\tilde{y}\right\rangle \\
& +\langle\epsilon(y-\tilde{y}), y-\tilde{y}\rangle \\
\leq & -\left\langle\mathbb{F}_{t^{*}}^{-1}(y-\tilde{y}), y-\tilde{y}\right\rangle+\langle\epsilon(y-\tilde{y}), y-\tilde{y}\rangle
\end{aligned}
$$

because of dissipativity of $-\Lambda_{f}$. Moreover, by coercivity of $\mathbb{F}_{t^{*}}^{-1}$ and sufficiently small $\epsilon>0$ it holds

$$
\begin{gathered}
\left\langle\left(\epsilon I-\mathbb{F}_{t^{*}}^{-1}-\Lambda_{f}\right)(y)-\left(\epsilon I-\mathbb{F}_{t^{*}}^{-1}-\Lambda_{f}\right)(\tilde{y}), y-\tilde{y}\right\rangle \\
\leq(-c+\epsilon)\|y-\tilde{y}\|^{2} \leq 0
\end{gathered}
$$

With the help of above lemmas we show that the nonlinear system $\Sigma^{f}$ is well-posed on $[0, \infty)$. We begin by showing that this holds on $\left[0, t^{*}\right]$. Here $t^{*}$ is the constant introduced in Assumption 2.1.

Lemma 3.3. Under Assumptions 2.1 and 2.2, the state trajectory of $\Sigma^{f}, x(t)$, and the output of $\Sigma^{f}, y$, exist in $X$ and in $L^{2}\left(\left[0, t^{*}\right] ; Y\right)$ respectively for $t<t^{*}$. Thus for every $x_{0} \in X$ and every $\nu \in L^{2}\left(\left[0, t^{*}\right] ; U\right)$ there exists a unique solution of (5) and (6). Moreover, $f(y) \in L^{2}\left(\left[0, t^{*}\right] ; U\right)$.

Proof. For the $t^{*}$ of Assumption 2.1, we start by proving the existence of $y \in L^{2}\left(\left[0, t^{*}\right) ; Y\right)$, the output of the closed-loop system. Consider the operator $-\Lambda_{f}$ on the domain $D\left(\Lambda_{f}\right)$ defined by $(13)$. It is easy to see that $D\left(\Lambda_{f}\right)$ is dense in $L^{2}\left(\left[0, t^{*}\right) ; Y\right)$, i.e. $\overline{D\left(\Lambda_{f}\right)}=L^{2}\left(\left[0, t^{*}\right) ; Y\right)$. Let us also define operator $\epsilon I-\mathbb{F}_{t^{*}}^{-1}$ on the domain $D(\epsilon I-$ $\left.\mathbb{F}_{t^{*}}^{-1}\right)=L^{2}\left(\left[0, t^{*}\right) ; Y\right)$. Notice that $\overline{D\left(\Lambda_{f}\right)}=D\left(\epsilon I-\mathbb{F}_{t^{*}}^{-1}\right)$ and that $\epsilon I-\mathbb{F}_{t^{*}}^{-1}$ is a continuous operator on $\overline{D\left(\Lambda_{f}\right)}$. By Lemma 3.1, operator $-\Lambda_{f}$ is $m$-dissipative. Moreover, $\epsilon I-\mathbb{F}_{t^{*}}^{-1}-\Lambda_{f}$ is dissipative by Lemma 3.2. Hence, $\epsilon I-\mathbb{F}_{t^{*}}^{-1}-\Lambda_{f}$ is $m$-dissipative by (Miyadera, 1992, Corollary 6.19). It means that for all $\lambda>0$,

$$
\operatorname{Ran}\left(\lambda I-\epsilon I+\mathbb{F}_{t^{*}}^{-1}+\Lambda_{f}\right)=L^{2}\left(\left[0, t^{*}\right) ; Y\right) .
$$

Taking $\lambda=\epsilon$, it shows that the equation

$$
\left(\Lambda_{f}+\mathbb{F}_{t^{*}}^{-1}\right)(y)=\omega
$$

has a unique solution $y \in D\left(\Lambda_{f}\right)$ for all $\omega \in L^{2}\left(\left[0, t^{*}\right) ; U\right)$. Choosing $\omega=\mathbb{F}_{t^{*}}^{-1} \Psi_{t^{*}} x_{0}+\nu \in L^{2}\left(\left[0, t^{*}\right) ; U\right)$, we find

$$
\left(\Lambda_{f}+\mathbb{F}_{t^{*}}^{-1}\right)(y)=\mathbb{F}_{t^{*}}^{-1} \Psi_{t^{*}} x_{0}+\nu
$$

which is equivalent to

$$
y=\Psi_{t^{*}} x_{0}+\mathbb{F}_{t^{*}} \nu-\mathbb{F}_{t^{*}} \Lambda_{f}(y)
$$

Hence, the output equation (16) has a unique solution $y \in L^{2}\left(\left[0, t^{*}\right) ; Y\right)$ for which $\Lambda_{f}(y) \in L^{2}\left(\left[0, t^{*}\right) ; U\right)$. The corresponding state trajectory, denoted by $x$, is obtained by injecting (16) in (5). Using (3), it follows from (16) that

$$
\begin{aligned}
\mathbf{P}_{t} y & =\mathbf{P}_{t} \Psi_{t^{*}} x_{0}+\mathbf{P}_{t} \mathbb{F}_{t^{*}} \nu-\mathbf{P}_{t} \mathbb{F}_{t^{*}} \Lambda_{f}(y) \\
& =\Psi_{t} x_{0}+\mathbb{F}_{t} \nu-\mathbb{F}_{t} \Lambda_{f}(y)
\end{aligned}
$$

for all $t<t^{*}$

For ease of reading, we will now (often) replace $\Lambda_{f}(y)$ by $f(y)$.

Lemma 3.4. Under Assumptions 2.1 and 2.2, for $t \leq$ $t^{*}$ the state and the output of the closed-loop system $\Sigma^{f}$ are continuously dependent on $x_{0}$ and on $\mathbf{P}_{t} \nu$. Moreover, there exist positive constants $\gamma_{i}, \quad i=1, \cdots, 4$ such that for all $t \leq t^{*}$ the following inequalities hold

$$
\begin{aligned}
\|x(t)-\tilde{x}(t)\| & \leq \gamma_{1}\left\|x_{0}-\tilde{x}_{0}\right\|+\gamma_{2}\left\|\mathbf{P}_{t} \nu-\mathbf{P}_{t} \tilde{\nu}\right\|, \\
\left\|\mathbf{P}_{t} y-\mathbf{P}_{t} \tilde{y}\right\| & \leq \gamma_{3}\left\|x_{0}-\tilde{x}_{0}\right\|+\gamma_{4}\left\|\mathbf{P}_{t} \nu-\mathbf{P}_{t} \tilde{\nu}\right\| .
\end{aligned}
$$


Proof. Consider two initial conditions $x_{0}$ and $\tilde{x}_{0} \in X$, two external inputs $\nu$ and $\tilde{\nu} \in L^{2}\left(\left[0, t^{*}\right] ; U\right)$ and $t<t^{*}$. The two corresponding state trajectories are given by

$$
\begin{aligned}
& x(t)=\mathbb{T}_{t} x_{0}+\Phi_{t} \nu-\Phi_{t} f(y) \\
& \tilde{x}(t)=\mathbb{T}_{t} \tilde{x}_{0}+\Phi_{t} \tilde{\nu}-\Phi_{t} f(\tilde{y})
\end{aligned}
$$

and the corresponding outputs are given by

$$
\begin{aligned}
& \mathbf{P}_{t} y=\Psi_{t} x_{0}+\mathbb{F}_{t} \nu-\mathbb{F}_{t} f(y) \\
& \mathbf{P}_{t} \tilde{y}=\Psi_{t} \tilde{x}_{0}+\mathbb{F}_{t} \tilde{\nu}-\mathbb{F}_{t} f(\tilde{y}) .
\end{aligned}
$$

We start by proving the continuous dependence for the output. From (19), it holds

$$
\begin{array}{r}
\mathbb{F}_{t}^{-1}\left(\mathbf{P}_{t} y-\mathbf{P}_{t} \tilde{y}\right)+\left(f\left(\mathbf{P}_{t} y\right)-f\left(\mathbf{P}_{t} \tilde{y}\right)\right) \\
=\mathbb{F}_{t}^{-1} \Psi_{t}\left(x_{0}-\tilde{x}_{0}\right)+\left(\mathbf{P}_{t} \nu-\mathbf{P}_{t} \tilde{\nu}\right),
\end{array}
$$

where the causality of $\mathbb{F}_{t}$ has been used, i.e., $\mathbb{F}_{t} f(y)=$ $\mathbb{F}_{t} f\left(\mathbf{P}_{t} y\right)$. Using the coercivity of $\mathbb{F}_{t}^{-1}$ and the inequality of $f\left(\right.$ or $\left.\Lambda_{f}\right)$, we find

$$
\begin{aligned}
& \left\langle\mathbb{F}_{t}^{-1}\left(\mathbf{P}_{t} y-\mathbf{P}_{t} \tilde{y}\right)+\left(f\left(\mathbf{P}_{t} y\right)-f\left(\mathbf{P}_{t} \tilde{y}\right)\right), \mathbf{P}_{t} y-\mathbf{P}_{t} \tilde{y}\right\rangle \\
& =\left\langle\mathbb{F}_{t}^{-1}\left(\mathbf{P}_{t} y-\mathbf{P}_{t} \tilde{y}\right), \mathbf{P}_{t} y-\mathbf{P}_{t} \tilde{y}\right\rangle \\
& +\left\langle f\left(\mathbf{P}_{t} y\right)-f\left(\mathbf{P}_{t} \tilde{y}\right), \mathbf{P}_{t} y-\mathbf{P}_{t} \tilde{y}\right\rangle \\
& \geq\left\langle\mathbb{F}_{t}^{-1}\left(\mathbf{P}_{t} y-\mathbf{P}_{t} \tilde{y}\right), \mathbf{P}_{t} y-\mathbf{P}_{t} \tilde{y}\right\rangle \\
& \geq c\left\|\mathbf{P}_{t} y-\mathbf{P}_{t} \tilde{y}\right\|^{2}
\end{aligned}
$$

for some $c>0$. Moreover, by the Cauchy-Schwarz inequality, we find

$$
\begin{aligned}
& \left\langle\mathbb{F}_{t}^{-1}\left(\mathbf{P}_{t} y-\mathbf{P}_{t} \tilde{y}\right)+\left(f\left(\mathbf{P}_{t} y\right)-f\left(\mathbf{P}_{t} \tilde{y}\right)\right), \mathbf{P}_{t} y-\mathbf{P}_{t} \tilde{y}\right\rangle \\
& \leq\left\|\mathbb{F}_{t}^{-1}\left(\mathbf{P}_{t} y-\mathbf{P}_{t} \tilde{y}\right)+f\left(\mathbf{P}_{t} y\right)-f\left(\mathbf{P}_{t} \tilde{y}\right)\right\| \cdot\left\|\mathbf{P}_{t} y-\mathbf{P}_{t} \tilde{y}\right\| .
\end{aligned}
$$

Combining (20), (21), and (22) yields

$$
\begin{aligned}
\| \mathbf{P}_{t} y & -\mathbf{P}_{t} \tilde{y}\left\|\leq \frac{1}{c}\right\| \mathbb{F}_{t}^{-1}\left(\mathbf{P}_{t} y-\mathbf{P}_{t} \tilde{y}\right)+f\left(\mathbf{P}_{t} y\right)-f\left(\mathbf{P}_{t} \tilde{y}\right) \| \\
& =\frac{1}{c}\left\|\mathbb{F}_{t}^{-1} \Psi_{t}\left(x_{0}-\tilde{x}_{0}\right)+\left(\mathbf{P}_{t} \nu-\mathbf{P}_{t} \tilde{\nu}\right)\right\| \\
& \leq \frac{\left\|\mathbb{F}_{t}^{-1}\right\| \cdot\left\|\Psi_{t}\right\|}{c}\left\|x_{0}-\tilde{x}_{0}\right\|+\frac{1}{c}\left\|\mathbf{P}_{t} \nu-\mathbf{P}_{t} \tilde{\nu}\right\|
\end{aligned}
$$

which is the second inequality of (17). Moreover, from (19)

$$
\begin{aligned}
& \left\|f\left(\mathbf{P}_{t} y\right)-f\left(\mathbf{P}_{t} \tilde{y}\right)\right\| \\
& =\left\|\mathbb{F}_{t}^{-1} \Psi_{t}\left(x_{0}-\tilde{x}_{0}\right)+\left(\mathbf{P}_{t} \nu-\mathbf{P}_{t} \tilde{\nu}\right)-\mathbb{F}_{t}^{-1}\left(\mathbf{P}_{t} y-\mathbf{P}_{t} \tilde{y}\right)\right\| \\
& \leq\left\|\mathbb{F}_{t}^{-1}\right\| \cdot\left\|\Psi_{t}\right\| \cdot\left\|x_{0}-\tilde{x}_{0}\right\|+ \\
& \quad\left\|\mathbf{P}_{t} \nu-\mathbf{P}_{t} \tilde{\nu}\right\|+\left\|\mathbb{F}_{t}^{-1}\right\| \cdot\left\|\mathbf{P}_{t} y-\mathbf{P}_{t} \tilde{y}\right\| .
\end{aligned}
$$

Using (23), it holds

$$
\begin{aligned}
& \left\|f\left(\mathbf{P}_{t} y\right)-f\left(\mathbf{P}_{t} \tilde{y}\right)\right\| \\
& \leq\left\|\mathbb{F}_{t}^{-1}\right\| \cdot\left\|\Psi_{t}\right\| \cdot\left\|x_{0}-\tilde{x}_{0}\right\|+\left\|\mathbf{P}_{t} \nu-\mathbf{P}_{t} \tilde{\nu}\right\|+ \\
& \left\|\mathbb{F}_{t}^{-1}\right\| \cdot\left[\frac{\left\|\mathbb{F}_{t}^{-1}\right\| \cdot\left\|\Psi_{t}\right\|}{c}\left\|x_{0}-\tilde{x}_{0}\right\|+\frac{1}{c}\left\|\mathbf{P}_{t} \nu-\mathbf{P}_{t} \tilde{\nu}\right\|\right] \\
& =\left\|\mathbb{F}_{t}^{-1}\right\| \cdot\left\|\Psi_{t}\right\| \cdot\left[1+\frac{\left\|\mathbb{F}_{t}^{-1}\right\|}{c}\right] \cdot\left\|x_{0}-\tilde{x}_{0}\right\|+ \\
& {\left[1+\frac{\left\|\mathbb{F}_{t}^{-1}\right\|}{c}\right] \cdot\left\|\mathbf{P}_{t} \nu-\mathbf{P}_{t} \tilde{\nu}\right\| .}
\end{aligned}
$$

Putting (18) and (24) together yields

$$
\begin{aligned}
& \|x(t)-\tilde{x}(t)\| \\
& \leq\left\|\mathbb{T}_{t}\right\| \cdot\left\|x_{0}-\tilde{x}_{0}\right\|+\left\|\Phi_{t}\right\| \cdot\left\|\mathbf{P}_{t} \nu-\mathbf{P}_{t} \tilde{\nu}\right\|+ \\
& \left\|\Phi_{t}\right\| \cdot\left\|f\left(\mathbf{P}_{t} y\right)-f\left(\mathbf{P}_{t} \tilde{y}\right)\right\| \\
& \leq\left\|\mathbb{T}_{t}\right\| \cdot\left\|x_{0}-\tilde{x}_{0}\right\|+\left\|\Phi_{t}\right\| \cdot\left[\left\|\mathbf{P}_{t} \nu-\mathbf{P}_{t} \tilde{\nu}\right\|+\right. \\
& \quad\left\|\mathbb{F}_{t}^{-1}\right\| \cdot\left\|\Psi_{t}\right\| \cdot\left(1+\frac{\left\|\mathbb{F}_{t}^{-1}\right\|}{c}\right) \cdot\left\|x_{0}-\tilde{x}_{0}\right\|+ \\
& \left.\quad\left(1+\frac{\left\|\mathbb{F}_{t}^{-1}\right\|}{c}\right) \cdot\left\|\mathbf{P}_{t} \nu-\mathbf{P}_{t} \tilde{\nu}\right\|\right] \\
& =\quad\left[\left\|\mathbb{T}_{t}\right\|+\left\|\Phi_{t}\right\| \cdot\left\|\mathbb{F}_{t}^{-1}\right\| \cdot\left\|\Psi_{t}\right\| \cdot\left(1+\frac{\left\|\mathbb{F}_{t}^{-1}\right\|}{c}\right)\right] . \\
& \quad\left\|x_{0}-\tilde{x}_{0}\right\|+\left\|\Phi_{t}\right\| \cdot\left(2+\frac{\left\|\mathbb{F}_{t}^{-1}\right\|}{c}\right) \cdot\left\|\mathbf{P}_{t} \nu-\mathbf{P}_{t} \tilde{\nu}\right\|
\end{aligned}
$$

which is the first inequality of (17).

We are ready now to prove the well-posedness of $\Sigma^{f}$.

Theorem 3.1. Under Assumptions 2.1 and 2.2, the system $\Sigma^{f}$ is well-posed in the sense of Definition 2.2 with $t_{f}=\infty$. Furthermore, inequalities, like (17) with $\gamma$ 's depending on $t$, hold for all $t>0$.

Proof. We prove this by induction. That is, we show that the system is well-posed on the interval $\left[0, k t^{*}\right]$, with $k \in \mathbb{N}$ and that inequalities, like (17) with $\gamma^{\prime}$ 's depending on $k$ hold. In Lemmas 3.3 and 3.4 we showed that this holds for $k=1$. Assuming now that it holds for $k=K$, we show the correctness for $k=K+1$. Let $x_{0} \in X$ and $\nu \in L^{2}\left(\left[0,(K+1) t^{*}\right] ; U\right)$ be given. For $t \in\left[0, K t^{*}\right]$ the assertion holds by the induction hypothesis, so we assume that $t \in\left(K t^{*},(K+1) t^{*}\right]$. We show first that we have a solution, and next we show the continuous dependence on the initial condition and external input.

By the induction hypothesis, the state and the output exist until $K t^{*}$, i.e.,

$$
\begin{aligned}
& x(t)=\mathbb{T}_{t} x_{0}+\Phi_{t} \nu-\Phi_{t} f(y) \\
& \mathbf{P}_{t} y=\Psi_{t} x_{0}+\mathbb{F}_{t} \nu-\mathbb{F}_{t} f(y)
\end{aligned}
$$

for $t \in\left[0, K t^{*}\right]$. For $\nu_{K}:=\left.\nu\right|_{\left[K t^{*},(K+1) t^{*}\right]} \in L^{2}\left(\left[0, t^{*}\right] ; U\right)$ and $\tau \in\left(0, t^{*}\right]$ we define

$$
\begin{aligned}
& x_{K}(\tau)=\mathbb{T}_{\tau} x\left(K t^{*}\right)+\Phi_{\tau} \nu_{K}-\Phi_{\tau} f\left(y_{K}\right) \\
& \mathbf{P}_{\tau} y_{K}=\Psi_{\tau} x\left(K t^{*}\right)+\mathbb{F}_{\tau} \nu_{K}-\mathbb{F}_{\tau} f\left(y_{K}\right) .
\end{aligned}
$$

Thus $x_{K}$ and $y_{K}$ are the state trajectory and the output generated by the initial condition $x\left(K t^{*}\right)$ and the external input $\nu_{K}$ in $\Sigma^{f}$. Again by the induction hypothesis this exists.

We extend the solutions $x$ and $y$ to the time interval $\left[K t^{*},(K+1) t^{*}\right]$ by defining

$$
\begin{aligned}
x\left(\tau+K t^{*}\right) & :=x_{K}(\tau) \\
\mathbf{P}_{\tau+K t^{*}} y & :=y \underset{K t^{*}}{\diamond} \mathbf{P}_{\tau} y_{K}
\end{aligned}
$$


for $\tau \in\left[0, t^{*}\right]$. Developing (26) for $\tau \in\left[0, t^{*}\right]$, we find

$$
\begin{aligned}
& x\left(\tau+K t^{*}\right)=\mathbb{T}_{\tau} x\left(K t^{*}\right)+\Phi_{\tau} \nu_{K}-\Phi_{\tau} f\left(y_{K}\right) \\
& =\mathbb{T}_{\tau}\left[\mathbb{T}_{K t^{*}} x_{0}+\Phi_{K t^{*}} \nu-\Phi_{K t^{*}} f(y)\right]+\Phi_{\tau} \nu_{K}-\Phi_{\tau} f\left(y_{K}\right) \\
& =\mathbb{T}_{\tau+K t^{*} x_{0}+\left(\mathbb{T}_{\tau} \Phi_{K t^{*}} \nu+\Phi_{\tau} \nu_{K}\right)-} \\
& \quad\left(\mathbb{T}_{\tau} \Phi_{K t^{*}} f(y)+\Phi_{\tau} f\left(y_{K}\right)\right) \\
& =\mathbb{T}_{\tau+K t^{*}} x_{0}+\Phi_{\tau+K t^{*}}\left(\underset{K t^{*}}{\diamond} \nu_{K}\right)-\Phi_{\tau+K t^{*}}\left(f(y) \underset{K t^{*}}{\diamond} f\left(y_{K}\right)\right) \\
& =\mathbb{T}_{\tau+K t^{*}} x_{0}+\Phi_{\tau+K t^{*}}(\nu)-\Phi_{\tau+K t^{*}}(f(y)),
\end{aligned}
$$

by the choice of $\nu_{K}$ and (27). This has the same form of (5). Looking at the output (27) for $\tau \in\left[0, t^{*}\right]$ yields

$$
\begin{aligned}
& \mathbf{P}_{\tau+K t^{*}} y=\mathbf{P}_{K t^{*}} y+\mathbf{S}_{K t^{*}} \mathbf{P}_{\tau} y_{K} \\
&=\Psi_{K t^{*}} x_{0}+\mathbb{F}_{K t^{*}} \nu-\mathbb{F}_{K t^{*}} f(y)+ \\
& \quad \mathbf{S}_{K t^{*}} \Psi_{\tau} x\left(K t^{*}\right)+\mathbf{S}_{K t^{*}} \mathbb{F}_{\tau} \nu_{K}-\mathbf{S}_{K t^{*}} \mathbb{F}_{\tau} f\left(y_{K}\right) \\
&=\Psi_{K t^{*}} x_{0}+\mathbb{F}_{K t^{*}} \nu-\mathbb{F}_{K t^{*}} f(y)+ \\
& \quad \mathbf{S}_{K t^{*}} \Psi_{\tau}\left[\mathbb{T}_{K t^{*}} x_{0}+\Phi_{K t^{*}} \nu-\Phi_{K t^{*}} f(y)\right]+ \\
& \quad \mathbf{S}_{K t^{*}} \mathbb{F}_{\tau} \nu_{K}-\mathbf{S}_{K t^{*}} \mathbb{F}_{t} f\left(y_{K}\right) \\
&=\left[\mathbf{P}_{K t^{*}} \Psi_{K t^{*}} x_{0}+\mathbf{S}_{K t^{*}} \Psi_{\tau} \mathbb{T}_{K t^{*}} x_{0}\right]+ \\
& {\left[\mathbf{S}_{K t^{*}}\left(\Psi_{\tau} \Phi_{K t^{*}} \nu+\mathbb{F}_{\tau} \nu_{K}\right)+\mathbf{P}_{K t^{*}} \mathbb{F}_{K t^{*}} \nu\right]-} \\
& {\left.\left[\mathbf{S}_{K t^{*}}\left(\Psi_{\tau} \Phi_{K t^{*}} f(y)+\mathbb{F}_{\tau} f y_{K}\right)\right)+\mathbf{P}_{K t^{*}} \mathbb{F}_{K t^{*}} f(y)\right] } \\
&= \Psi_{\tau+K t^{*}} x_{0}+\mathbb{F}_{\tau+K t^{*}}\left(\nu \diamond_{K t^{*}} \nu_{K}\right)-\mathbb{F}_{\tau+K t^{*}}\left(f(y) \diamond f\left(y_{K}\right)\right) \\
&= \Psi_{\tau+K t^{*}} x_{0}+\mathbb{F}_{\tau+K t^{*}}(\nu)-\mathbb{F}_{\tau+K t^{*}}(f(y)),
\end{aligned}
$$

using the definition of $\nu_{K}$ and (27). This has the same form of (6) which means that the solution can be extended on $\left[0,(K+1) t^{*}\right]$.

It remains to show that an estimate like (17) holds on the extended time interval. Since the proof for the state and the output are very similar we only show it for the state. Let $x$ and $\tilde{x}$ denote the two states. Since by the induction hypothesis we have the estimate for $t \in\left[0, K t^{*}\right]$, we take $t=\tau+K t^{*}$ with $\tau \in\left(0, t^{*}\right]$. By (26) we have that $x\left(\tau+K t^{*}\right)=x_{K}(\tau)$ and the same for $\tilde{x}$. So using the induction hypothesis (twice) we obtain

$$
\begin{aligned}
& \left\|x\left(\tau+K t^{*}\right)-\tilde{x}\left(\tau+K t^{*}\right)\right\|=\left\|x_{K}(\tau)-\tilde{x}_{K}(\tau)\right\| \\
& \leq \gamma_{1}\left\|x\left(K t^{*}\right)-\tilde{x}\left(K t^{*}\right)\right\|+\gamma_{2}\left\|\mathbf{P}_{\tau} \nu_{K}-\mathbf{P}_{\tau} \tilde{\nu}_{K}\right\| \\
& \leq \gamma_{1}\left[\gamma_{1, K}\left\|x_{0}-\tilde{x}_{0}\right\|+\gamma_{2, K}\left\|\mathbf{P}_{K t^{*}} \nu-\mathbf{P}_{K t^{*}} \tilde{\nu}\right\|\right]+ \\
& \quad \gamma_{2}\left\|\mathbf{P}_{\tau} \nu_{K}-\mathbf{P}_{\tau} \tilde{\nu}_{K}\right\| \\
& =\gamma_{1} \gamma_{1, K}\left\|x_{0}-\tilde{x}_{0}\right\|+ \\
& \quad \gamma_{1} \gamma_{2, K}\left\|\mathbf{P}_{K t^{*}} \nu-\mathbf{P}_{K t^{*}}\right\|+\gamma_{2}\left\|\mathbf{P}_{\tau} \nu_{K}-\mathbf{P}_{\tau} \tilde{\nu}_{K}\right\| \\
& \leq \gamma_{1, K+1}\left\|x_{0}-\tilde{x}_{0}\right\|+\gamma_{2, K+1}\left\|\mathbf{P}_{(K+1) t^{*}}-\mathbf{P}_{(K+1) t^{*}}\right\|
\end{aligned}
$$

for some $\gamma_{1, K+1}$ and $\gamma_{2, K+1}$, where we have used the definition of $\nu_{K}$ once more.

\section{Application to linear port-Hamiltonian systems}

In this section we apply Theorem 3.1 to a particular class of linear port-Hamiltonian systems. Consider firstorder linear port-Hamiltonian systems described by the following PDE :

$$
\frac{\partial x}{\partial t}(\zeta, t)=P_{1} \frac{\partial}{\partial \zeta}(\mathcal{H}(\zeta) x(\zeta, t))+P_{0}(\mathcal{H}(\zeta) x(\zeta, t)),
$$

with boundary control, conditions and observation

$$
\begin{aligned}
u(t) & =W_{B, 1}\left[\begin{array}{l}
\mathcal{H}(b) x(b, t) \\
\mathcal{H}(a) x(a, t)
\end{array}\right], \\
0 & =W_{B, 2}\left[\begin{array}{l}
\mathcal{H}(b) x(b, t) \\
\mathcal{H}(a) x(a, t)
\end{array}\right], \\
y(t) & =W_{C}\left[\begin{array}{l}
\mathcal{H}(b) x(b, t) \\
\mathcal{H}(a) x(a, t)
\end{array}\right],
\end{aligned}
$$

where $\zeta \in[a, b], t \geq 0, x(\zeta, t) \in \mathbb{R}^{n}, P_{1} \in \mathbb{R}^{n \times n}$ is invertible and self-adjoint, $P_{0} \in \mathbb{R}^{n \times n}$ is skew-adjoint, $\mathcal{H} \in$ $L^{\infty}\left([a, b] ; \mathbb{R}^{n \times n}\right)$ such that $\mathcal{H}(\zeta)=\mathcal{H}^{*}(\zeta)$ and $m I \leq \mathcal{H}(\zeta)$ for a.e. $\zeta$ and constant $m>0$ independent of $\zeta$, see $(\mathrm{Ja}-$ cob and Zwart, 2012, Definition 7.1.2). Furthermore, we assume that $y(t), u(t) \in \mathbb{R}^{k}$, and $\operatorname{rank}\left[\begin{array}{l}W_{B, 1} \\ W_{B, 2}\end{array}\right]=n$. The above implies that the operator associated to the homogeneous port-Hamiltonian system, i.e., (28)-(29) with $u \equiv 0$, generates a contraction semigroup on the state space $X$. Here $X$ is $L^{2}\left([a, b] ; \mathbb{R}^{n}\right)$ equipped with the inner product

$$
\langle f, g\rangle_{X}=\int_{a}^{b} f(\zeta)^{*} \mathcal{H}(\zeta) g(\zeta) d \zeta
$$

Furthermore, it follows by (Jacob and Zwart, 2012, Theorem 11.3.2) that (28)-(29) is a boundary control system in the sense of (Jacob and Zwart, 2012, Definition 11.1.1).

The energy associated to (28) is given by $E(t)=$ $\frac{1}{2}\|x(t)\|_{X}^{2}$. Along classical solutions of (28), an expression of the time derivative of the energy is provided in (Jacob and Zwart, 2012, Theorem 7.1.5) and is given by

$$
\frac{d E}{d t}(t)=\frac{1}{2}\left[(\mathcal{H}(\zeta) x(\zeta, t))^{T} P_{1} \mathcal{H}(\zeta) x(\zeta, t)\right]_{a}^{b} .
$$

We suppose that (28)-(29) is impedance passive, i.e., that $\frac{d E}{d t}(t) \leq u^{T}(t) y(t)$ holds along classical solutions.

Lemma 4.1. Let us consider the impedance passive boundary control system (28)-(29). Assume that $P_{1} \mathcal{H}(\zeta)$ is diagonalizable, i.e., there exist $\Delta(\zeta)$, a diagonal matrixvalued function and $S(\zeta)$, a matrix-valued function, both continuously differentiable on $[a, b]$ such that

$$
P_{1} \mathcal{H}(\zeta)=S^{-1}(\zeta) \Delta(\zeta) S(\zeta), \quad \zeta \in[a, b]
$$

Furthermore, assume that

$$
\operatorname{rank}\left[\begin{array}{c}
W_{B, 1} \\
W_{B, 2} \\
W_{C}
\end{array}\right]=n+\operatorname{rank}\left(W_{C}\right) .
$$

Then, the system (28)-(29) is regular, well-posed and satisfies $^{3} \lim _{\mathfrak{R} \mathfrak{e}(s) \rightarrow \infty} G(s)=\lim _{s \rightarrow \infty, s \in \mathbb{R}} G(s)=: \mathcal{D}$ where $G(s)$ is the transfer function of (28)-(29). Moreover, the feed-through term $\mathcal{D}$ is coercive.

\footnotetext{
${ }^{3}$ Note that the convergence is uniform with respect to the imaginary part of $s$, see (Jacob and Zwart, 2012, Lemmas 13.2.6, 13.2.7, Theorem 13.3.1).
} 
Proof. The regularity and the well-posedness are provided by (Jacob and Zwart, 2012, Theorem 13.2.2).

By (Jacob and Zwart, 2012, Lemma 13.2.5), the diagonal matrix $\Delta(\zeta)$ has the form

$$
\Delta(\zeta)=\left[\begin{array}{cc}
\Lambda(\zeta) & 0 \\
0 & \Theta(\zeta)
\end{array}\right]
$$

where $\Lambda(\zeta)$ is a diagonal real matrix-valued function with strictly positive functions on the diagonal and $\Theta(\zeta)$ is a diagonal real matrix-valued function with strictly negative functions on the diagonal.

We consider the state transformation

$$
z(\zeta, t)=\left[\begin{array}{c}
z_{+}(\zeta, t) \\
z_{-}(\zeta, t)
\end{array}\right]:=S(\zeta) x(\zeta, t)
$$

In this way, the PDE (28) becomes

$$
\begin{aligned}
\frac{\partial z}{\partial t}(\zeta, t)= & \frac{\partial}{\partial \zeta}(\Delta z)(\zeta, t)+S(\zeta) \frac{d S^{-1}(\zeta)}{d \zeta} \Delta(\zeta) z(\zeta, t) \\
& +S(\zeta) P_{0} S^{-1}(\zeta) z(\zeta, t)
\end{aligned}
$$

and (29) becomes

$$
\left[\begin{array}{c}
0 \\
u(t)
\end{array}\right]=K u_{s}(t)+Q y_{s}(t), y(t)=O_{1} u_{s}(t)+O_{2} y_{s}(t)
$$

where

$$
u_{s}(t)=\left[\begin{array}{c}
\Lambda(b) z_{+}(b, t) \\
\Theta(a) z_{-}(a, t)
\end{array}\right], y_{s}(t)=\left[\begin{array}{c}
\Lambda(a) z_{+}(a, t) \\
\Theta(b) z_{-}(b, t)
\end{array}\right] .
$$

$K$ and $Q$ are two square $n \times n$ matrices with [ $K Q$ ] of rank $n$ and $O_{1}$ and $O_{2}$ are $k \times n$ matrices, see (Jacob and Zwart, 2012, Section 13.4). By (Jacob and Zwart, 2012, Lemma 13.1.14), the limit of the transfer function of (36)-(37) for $\mathfrak{R e}(s) \rightarrow \infty$ is equal to the same limit of the transfer function of

$$
\frac{\partial z}{\partial t}(\zeta, t)=\frac{\partial}{\partial \zeta}(\Delta z)(\zeta, t)
$$

with the boundary input and output (37). If we write $O_{1} K^{-1}$ as $[\star \mathcal{D}]$, with $\mathcal{D} k \times k$, then by (Jacob and Zwart, 2012, Theorem 13.3.1), this $\mathcal{D}$ is the feedthrough operator of our system, i.e., $\lim _{\mathfrak{R} \mathfrak{e}(s) \rightarrow \infty} G(s)=\lim _{s \rightarrow \infty, s \in \mathbb{R}} G(s)=$ $\mathcal{D}$. Since the system is impedance passive, the transfer function is positive real, see e.g. (Jacob and Zwart, 2012, Example 12.2.3). Hence $\mathcal{D}$ satisfies $\mathcal{D}+\mathcal{D}^{T} \geq 0$. To prove that this inequality is strict, we begin by showing that $\mathcal{D}$ is invertible. Suppose by contradiction that $\mathcal{D}$ is not invertible. By the relation with $O_{1} K^{-1}$ this implies that there exists a non-zero $u \in \mathbb{R}^{k}$ such that $O_{1} K^{-1}\left[\begin{array}{l}0 \\ u\end{array}\right]=0$. Let us define

$$
\left[\begin{array}{l}
\Lambda(b) z_{+}(b) \\
\Theta(a) z_{-}(a)
\end{array}\right]:=K^{-1}\left[\begin{array}{l}
0 \\
u
\end{array}\right],\left[\begin{array}{c}
z_{+}(a) \\
z_{-}(b)
\end{array}\right]:=\left[\begin{array}{l}
0 \\
0
\end{array}\right] .
$$

In this way,

$$
y=O_{1} u_{s}(t)+O_{2} u_{s}(t)=O_{1} K^{-1}\left[\begin{array}{l}
0 \\
u
\end{array}\right]+0=0 .
$$

It can be shown that the energy balance combined with the impedance passivity gives

$$
\frac{1}{2}\left[z^{T}(b) \mathcal{H}_{S}(b) \Delta(b) z(b)-z^{T}(a) \mathcal{H}_{S}(a) \Delta(a) z(a)\right] \leq u^{T} y,
$$

where $\mathcal{H}_{S}(\zeta)=S^{-T}(\zeta) \mathcal{H}(\zeta) S^{-1}(\zeta)$. Using (40) and (41), (42) gives

$$
z_{+}^{T}(b) \mathcal{H}_{11}(b) \Lambda(b) z_{+}(b)-z_{-}^{T}(a) \mathcal{H}_{22}(a) \Theta(a) z_{-}(a) \leq 0,
$$

where the decomposition $H_{S}(\zeta)=\left[\begin{array}{ll}\mathcal{H}_{11}(\zeta) & \mathcal{H}_{12}(\zeta) \\ \mathcal{H}_{21}(\zeta) & \mathcal{H}_{22}(\zeta)\end{array}\right]$ has been used.

Since $\mathcal{H}_{11}(\zeta)$ and $\mathcal{H}_{22}(\zeta)$ are two principal matrices of $\mathcal{H}_{S}(\zeta)$, since $\mathcal{H}_{11}(\zeta) \Lambda(\zeta)=\Lambda(\zeta) \mathcal{H}_{11}(\zeta), \mathcal{H}_{22}(\zeta) \Theta(\zeta)=$ $\Theta(\zeta) \mathcal{H}_{22}(\zeta)$, and since $\mathcal{H}_{S}(\zeta)$ is positive definite, the relations

$$
\mathcal{H}_{11}(\zeta) \Lambda(\zeta)>0, \mathcal{H}_{22}(\zeta) \Theta(\zeta) \quad<0
$$

hold. Combining this with (43) implies that $z_{+}(b)=0$ and $z_{-}(a)=0$. Using (40), this yields $K^{-1}\left[\begin{array}{l}0 \\ u\end{array}\right]=0$. This means that $u$ is identically 0 , which is a contradiction. Hence, $\mathcal{D}$ is invertible. So we have shown the invertibility of the feedthrough matrix for any impedance passive port-Hamiltonian system for which the Hamiltonian can be written as (32). If we put all inputs except the first one equal to zero and we only consider the first output, then this scalar input-output system is impedance passive. The above result implies that its feedthrough is invertible. It is easy to see that this (new) feedthrough equals $\mathcal{D}_{11}$. We can repeat this argument for all components of the input vector, and so we find that $\mathcal{D}_{i i} \neq 0$ for $i=1, \cdots, k$. Since the system is impedance passive, we even know that $\mathcal{D}_{i i}>0$ for $i=1, \cdots, k$.

Let $\mathcal{U}$ be a unitary $k \times k$ matrix, and define $\tilde{u}=\mathcal{U} u$ and $\tilde{y}=\mathcal{U} y$. Then $\tilde{u}^{T} \tilde{y}=u^{T} y$, and so the port-Hamiltonian system with the new input $\tilde{u}$ and new output $\tilde{y}$ is still impedance passive. The feedthrough matrix $\tilde{\mathcal{D}}$ of this system is related to $\mathcal{D}$ via

$$
\tilde{\mathcal{D}}=\mathcal{U} \mathcal{D} \mathcal{U}^{T}
$$

Since the port-Hamiltonian system with input $\tilde{u}$ and output $\tilde{y}$ still satisfies all the assumptions, we have that the diagonal elements of $\tilde{\mathcal{D}}$ are strictly positive.

Assume now that $\mathcal{D}$ is not coercive. Thus there exists a non-zero $u_{0} \in \mathbb{R}^{k}$ such that $u_{0}^{T} \mathcal{D} u_{0}=0$. Without loss of generality, we may assume that $u_{0}$ has norm one. Let $\mathcal{U}$ be a unitary matrix which maps this vector onto $[1,0, \cdots, 0]^{T}$. Then

$$
0=u_{0}^{T} \mathcal{D} u_{0}=\left[\begin{array}{lll}
1 & 0 & 0
\end{array}\right] \mathcal{U} \mathcal{D} \mathcal{U}^{T}\left[\begin{array}{l}
1 \\
0 \\
0
\end{array}\right]=\tilde{\mathcal{D}}_{11}
$$

This is a contradiction, and so $\mathcal{D}$ is coercive.

The following theorem characterizes closed-loop systems that result of the interconnection of a linear system comprises in the class of linear port-Hamiltonian systems introduced in this section with a static nonlinearity that satisfies Assumption 2.2.

Theorem 4.1. Consider the first order port-Hamiltonian system described by (28)-(29) that satisfies the assumptions of Lemma 4.1. Furthermore, consider the interconnection $u(t)=-f(y(t))$ where $f(\cdot)$ is a nonlinear function 
that satisfies Assumption 2.2. Then, the resulting nonlinear system is well-posed.

Proof. By Lemma 4.1, the linear system is well-posed, regular and the corresponding feedthrough operator, $\mathcal{D}$, is coercive. Moreover, since

$$
\lim _{\mathfrak{R e}(s) \rightarrow \infty} G(s)=\mathcal{D},
$$

there exists a sufficiently large $\alpha \in \mathbb{R}$ such that $G(s)$ is boundedly invertible on $\mathbb{C}_{\alpha}:=\{s \in \mathbb{C} \mid \mathfrak{R e}(s)>\alpha\}$. By the Paley-Wiener Theorem, see e.g. (Jacob and Zwart, 2012, Theorem A.2.9), there exists a sufficiently small $t^{*}>0$ such that the operator $\mathbb{F}_{t}$ is boundedly invertible for all $t<t^{*}$. Moreover, since the port-Hamiltonian system (28)-(29) is impedance passive, the operator $\mathbb{F}_{t}$ is positive, i.e., along any solution on $\left[0, t^{*}\right)$ it holds $\left\langle\mathbb{F}_{t} u, u\right\rangle \geq 0$. This fact together with the invertibility, implies coercitivity of the operator $\mathbb{F}_{t}$ for $t<t^{*}$, i.e. Assumption 2.1 is satisfied.

Since the considered nonlinearity satisfies Assumption 2.2 , Theorem 3.1 provides the well-posedness of the closedloop system.

\section{Example : The vibrating string with a nonlinear damper at the boundary}

In this section, Theorem 4.1 is illustrated with a vibrating string with a nonlinear damper attached to it. This system can be described by means of the following PDE

$$
\begin{aligned}
& \frac{\partial^{2} w}{\partial t^{2}}(\zeta, t)=\frac{1}{\rho(\zeta)} \frac{\partial}{\partial \zeta}\left(T(\zeta) \frac{\partial w}{\partial \zeta}(\zeta, t)\right), w(\zeta, 0)=w_{0}(\zeta) \\
& -f\left(\frac{\partial w}{\partial t}(1, t)\right)=T(1) \frac{\partial w}{\partial \zeta}(1, t), \frac{\partial w}{\partial t}(0, t)=0
\end{aligned}
$$

where $\zeta \in[0,1]$ is the spatial variable, $w(\zeta, t)$ is the vertical position of the string at position $\zeta$ and at time $t, T(\zeta)$ and $\rho(\zeta)$ represent the Young's modulus and the mass density respectively and are supposed to be positive, continuously differentiable functions. Equation (46) can be seen as the linear PDE

$$
\frac{\partial^{2} w}{\partial t^{2}}(\zeta, t)=\frac{1}{\rho(\zeta)} \frac{\partial}{\partial \zeta}\left(T(\zeta) \frac{\partial w}{\partial \zeta}(\zeta, t)\right),
$$

with boundary input and output

$$
u(t)=T(1) \frac{\partial w}{\partial \zeta}(1, t), \quad y(t)=\frac{\partial w}{\partial t}(1, t)
$$

connected by the nonlinear feedback $u(t)=-f(y(t))$. Defining the state variables $x_{1}(\zeta, t)=\rho \frac{\partial w}{\partial t}$ (the momentum) and $x_{2}(\zeta, t)=\frac{\partial w}{\partial \zeta}$ (the strain), the linear PDE (47) admits a port-Hamiltonian representation in the form

$$
\frac{\partial x}{\partial t}(\zeta, t)=P_{1} \frac{\partial}{\partial \zeta}(\mathcal{H}(\zeta) x(\zeta, t))
$$

where $x(\zeta, t)=\left[x_{1}(\zeta, t) \quad x_{2}(\zeta, t)\right]^{T}, P_{1}=\left[\begin{array}{ll}0 & 1 \\ 1 & 0\end{array}\right]$ and $\mathcal{H}(\zeta)=\left[\begin{array}{cc}\frac{1}{\rho(\zeta)} & 0 \\ 0 & T(\zeta)\end{array}\right]$. This PDE falls in the well-established class of linear port-Hamiltonian systems on 1-D spatial domain, whose properties are considered in the previous section. For this system, $P_{1} \mathcal{H}(\zeta)$ can be expressed as $P_{1} \mathcal{H}(\zeta)=S^{-1}(\zeta) \Delta(\zeta) S(\zeta)$ where

$$
S(\zeta)=\left[\begin{array}{cc}
\frac{1}{2 \gamma(\zeta)} & \frac{\rho(\zeta)}{2} \\
-\frac{1}{2 \gamma(\zeta)} & \frac{\rho(\zeta)}{2}
\end{array}\right] \text { and } \Delta(\zeta)=\left[\begin{array}{cc}
\Lambda(\zeta) & 0 \\
0 & -\Theta(\zeta)
\end{array}\right]
$$

with $\Lambda(\zeta)=\Theta(\zeta)=\gamma(\zeta)=\sqrt{\frac{T(\zeta)}{\rho(\zeta)}}$. Then Theorem 4.1 establishes well-posedness of (46) for any function $f(\cdot)$ that satisfies Assumption 2.2, e.g. $f(y)=y^{3}$ or any odd polynomial representing nonlinear damping at the end of the string.

\section{Conclusion and future work}

In this paper, well-posedness of a class of infinitedimensional linear systems interconnected with a static nonlinearity has been proven. The problem has been introduced with a simple (counter) example. As main result, sufficient conditions on the linear system to end up with a well-posed closed-loop system are provided, extending the class of admissible nonlinearities presented in (Tucsnak and Weiss, 2014). Moreover, it is shown that impedance passive port-Hamiltonian systems satisfy the necessary conditions of the well-posed linear system. Finally, the result has been applied on a vibrating string with a nonlinear damper at the boundary.

Future work aims at extending the class of nonlinearities for which a closed-loop system is well-posed to dynamical systems.

\section{References}

Augner, B., 2016. Well-posedness and stability of linear portHamiltonian systems with nonlinear boundary feedback. SIAM Journal on Control and Optimization (submitted for publication).

Curtain, R., Zwart, H., 1995. An Introduction to Infinite Dimensional Linear Systems Theory. Springer Verlag, Germany.

Jacob, B., Zwart, H., 2012. Linear Port-Hamiltonian Systems on Infinite-dimensional Spaces. No. 223 in Operator Theory: Advances and Applications. Springer Verlag, 10.1007/978-3-03480399-1.

Miyadera, I., 1992. Nonlinear Semigroups. Translations of Mathematical Monographs. Amer Mathematical Society.

Staffans, O., 2005. Well-Posed Linear Systems. Encyclopedia of Mathematics and its Applications. Cambridge University Press.

Tucsnak, M., Weiss, G., 2009. Observation and control for operator semigroups. Birkhäuser Verlag, Basel.

Tucsnak, M., Weiss, G., 2014. Well-posed systems - The LTI case and beyond. Automatica 50 (7), 1757 - 1779.

Zwart, H., Le Gorrec, Y., Maschke, B., January 2013. Using System Theory to prove Existence of Non-Linear PDE's. In: 1er IFAC Workshop Control of Systems Governed by Partial Differential Equations, CPDE'13. France, pp. 1-3. 